\title{
A NOTE ON THE DZIOBEK CENTRAL CONFIGURATIONS
}

\author{
JAUME LLIBRE
}

(Communicated by Yingfei Yi)

\begin{abstract}
For the Newtonian $n$-body problem in $\mathbb{R}^{n-2}$ with $n \geq 3$ we prove that the following two statements are equivalent.

(a) Let $x$ be a Dziobek central configuration having one mass located at the center of mass.

(b) Let $x$ be a central configuration formed by $n-1$ equal masses located at the vertices of a regular $(n-2)$-simplex together with an arbitrary mass located at its barycenter.
\end{abstract}

\section{InTRODUCTION AND STATEMENT OF THE MAIN RESUlts}

The main problem of the classical Celestial Mechanics is the $n$-body problem; i.e. the description of the motion of $n$ particles of positive masses under their mutual Newtonian gravitational forces. This problem is completely solved only when $n=2$, and for $n>2$ there are only few partial results.

The equations of motion of the Newtonian $n$-body problem in the $d$-dimensional space $\mathbb{R}^{d}$ are

$$
\ddot{x}_{i}=\sum_{j=1, j \neq i}^{n} \frac{m_{j}\left(x_{j}-x_{i}\right)}{r_{i j}^{3}}, \text { for } i=1, \ldots, n,
$$

where $x_{i} \in \mathbb{R}^{d}$ are the positions of the bodies, $r_{i j}=\left|x_{i}-x_{j}\right|$ are their mutual distances, and $m_{i}$ are their masses.

The vector $x=\left(x_{1}, \ldots, x_{n}\right) \in \mathbb{R}^{n d}$ denotes the configuration of the system formed by the $n$ bodies. Clearly the differential equations of motion are well-defined when the configuration is of non-collision, that is when $r_{i j} \neq 0$ for $i \neq j$.

The dimension $\delta(x)$ of a configuration $x$ is defined as the dimension of the smallest affine subspace of $\mathbb{R}^{d}$ which contains all of the points $x_{i}$. Of course, the configurations with $\delta(x)=1,2,3$ are called collinear, planar and spatial, respectively. The dimension $\delta(x)$ of a non-collision configuration of $n \geq 2$ bodies satisfies $1 \leq \delta(x) \leq n-1$.

The total mass and the center of mass of the $n$ bodies are defined as

$$
M=m_{1}+\ldots+m_{n}, \quad c=\frac{1}{M}\left(m_{1} x_{1}+\cdots+m_{n} x_{n}\right),
$$

Received by the editors November 27, 2011 and, in revised form, March 16, 2014.

2010 Mathematics Subject Classification. Primary 70F07; Secondary 70F15.

Key words and phrases. Central configuration, $n$-body problem.

The first author is partially supported by a MINECO/FEDER grant MTM2008-03437 and MTM2013-40998-P, an AGAUR grant number 2014SGR-568, an ICREA Academia, the grants FP7-PEOPLE-2012-IRSES 318999 and 316338, grant UNAB13-4E-1604. 
respectively. A configuration $x$ is a central configuration if the acceleration vectors of the bodies satisfy

$$
\sum_{j=1, j \neq i}^{n} \frac{m_{j}\left(x_{j}-x_{i}\right)}{r_{i j}^{3}}+\lambda\left(x_{i}-c\right)=0, \quad \text { for } \quad i=1, \ldots, n .
$$

Central configurations started to be studied in the second part of the 18th century, and there is extensive literature concerning these solutions. For a classical background, see the sections on central configurations in the books of Wintner [18] and Hagihara [7. For a modern background see, for instance, the papers of Albouy and Chenciner [3, Albouy and Kaloshin 44, Hampton and Moeckel [8, Moeckel [10], Palmore [14], Saari [15, Schmidt [16], Xia [19],... One of the reasons why central configurations are important is that they allow us to obtain the unique explicit solutions in function of the time of the $n$-body problem known until now, as the homographic solutions for which the ratios of the mutual distances between the bodies remain constant. They are also important because the total collision or the total parabolic escape at infinity in the $n$-body problem is asymptotic to central configurations, see for more details Dziobek [6] and [15. Also if we fix the total energy $h$ and the angular momentum $c$ of the $n$-body problem, then some of the bifurcation points $(h, c)$ for the topology of the level sets with energy $h$ and angular momentum $c$ are related with the central configurations, see Meyer [12] and Smale [17] for a full background on these topics.

Moulton [13] proved that for a fixed mass vector $m=\left(m_{1}, \ldots, m_{n}\right)$ and a fixed ordering of the bodies along the line, there exists a unique collinear central configuration, up to translation and scaling.

At the other extreme of the dimension range, Lagrange 9] showed that for $n=3$, the only central configuration $x$ with $\delta(x)=n-1=2$ is the equilateral triangle, and it is central for all choices of the masses. An analogous result of Lagrange's result holds for all $n$. Thus, it is well known that for $n \geq 3$ the only central configuration $x$ with $\delta(x)=n-1$ of the $n$-body problem is formed by the vertices of a regular $(n-1)$-simplex, which is central for all choices of the masses. Of course, a 1-simplex is a closed interval, a 2-simplex is a closed equilateral triangle, a 3-simplex is a closed regular tetrahedron, and so on.

For other values of the dimension $\delta(x)$ the problem of finding or even counting the central configurations $x$ of the $n$-body problem is very difficult, see for instance Moeckel [11. The dimension $\delta(x)=2$ is of course the most interesting of all, because planar central configurations give rise to physically realistic periodic orbits. For $n=4$, Dziobek [6] formulated the planar central configuration problem in terms of mutual distances $r_{i j}$ and obtained algebraic equations characterizing the central configurations. His approach has been adopted and developed by Albouy [1,2] in his study of the central configurations with four equal masses, see also Albouy and Llibre [5. The natural generalization to higher $n$ is the case $\delta(x)=n-2$. Following [1] and [11] we call such central configurations Dziobek configurations.

The goal of this paper is to prove the following result.

Theorem 1. The following two statements are equivalent for the $n$-body problem with $n \geq 3$.

(a) Let $x$ be a central configuration with $\delta(x)=n-2$ (i.e. a Dziobek central configuration) having one mass located at the center of mass. 
(b) Let $x$ be a central configuration formed by $n-1$ equal masses located at the vertices of a regular $(n-2)$-simplex together with an arbitrary mass located at its barycenter.

Of course, statement (b) of Theorem 1 implies immediately statement (a). The converse implication is proved in section 3 .

If the central configuration is not Dziobek, then the equivalence of Theorem 1 does not hold. Thus, for instance, consider a regular polygon with $n-1>3$ equal masses located at its vertices and an arbitrary mass located at its barycenter. This is a non-Dziobek central configuration having one mass located at the center of mass, and since $n>4$ it is different from the configuration formed by the vertices of a regular $(n-2)$-simplex together with its barycenter.

\section{Equations for the Dziobek Central Configurations}

Since we want to study the Dziobek central configurations, we consider the $n$ body problem in $\mathbb{R}^{n-2}$. To each configuration $x=\left(x_{1}, \ldots, x_{n}\right) \in \mathbb{R}^{n(n-2)}$ we associate the $n \times n$ matrix

$$
X=\left(\begin{array}{ccc}
1 & \cdots & 1 \\
x_{1} & \cdots & x_{n} \\
0 & \cdots & 0
\end{array}\right)
$$

Let $X_{k}$ be the $(n-1) \times(n-1)$ matrix obtained deleting from the matrix $X$ its $k$-th column and its last row. Then define $\Delta_{k}=(-1)^{k+1} \operatorname{det}\left(X_{k}\right)$ for $k=1, \ldots, n$.

Dziobek [6] (see also equations (8) and (16) of Moeckel [1]) reduces the equations for the central configurations (11) of the $n$-body problem to the following system of

$$
N=\frac{n(n-1)}{2}+n+2
$$

equations and $N$ unknowns:

$$
\begin{aligned}
& \frac{1}{r_{i j}^{3}}=c_{1}+c_{2} \frac{\Delta_{i} \Delta_{j}}{m_{i} m_{j}} \\
& t_{i}-t_{j}=0
\end{aligned}
$$

for $1 \leq i<j \leq n$, with

$$
t_{i}=\sum_{j=1, j \neq i}^{n} \Delta_{j} r_{i j}^{2} .
$$

The $N$ unknowns in equations (2) are the $n(n-1) / 2$ mutual distances $r_{i j}$, the $n$ variables $\Delta_{i}$, and the two constants $c_{k}$.

\section{Proof of Theorem 1}

In order to complete the proof of Theorem 1 we must prove that statement (a) of that theorem implies statement (b).

We assume that $x=\left(x_{1}, \ldots, x_{n}\right) \in \mathbb{R}^{n(n-2)}$ is a Dziobek central configuration having one mass located at the center of masses. Without loss of generality we can suppose:

(i) the center of mass is at the origin of coordinates;

(ii) the mass $m_{n}$ is located at the center of mass, i.e. $x_{n}=0$; 
(iii) the unit of mass is taken in such a way that $m_{n-1}=1$. Then

$$
x_{n-1}=-\sum_{i=1}^{n-2} m_{i} x_{i} .
$$

Since $x$ is a Dziobek central configuration it satisfies (2). Easy computations using the properties of the determinants show that

$$
\begin{aligned}
& \frac{\Delta_{i} \Delta_{j}}{m_{i} m_{j}}=\operatorname{det}\left(x_{1} \cdots x_{n-2}\right)^{2} \quad \text { for } \quad 1 \leq i<j \leq n-1, \\
& \frac{\Delta_{i} \Delta_{n}}{m_{i} m_{n}}=\frac{M-m_{n}}{m_{n}} \operatorname{det}\left(x_{1} \cdots x_{n-2}\right)^{2} \quad \text { for } \quad i=1, \ldots, n-1 .
\end{aligned}
$$

Then, the first equations of (2) become

$$
\begin{aligned}
& \frac{1}{r_{i j}^{3}}=c_{1}+c_{2} \operatorname{det}\left(x_{1} \cdots x_{n-2}\right)^{2} \quad \text { for } \quad 1 \leq i<j \leq n-1, \\
& \frac{1}{r_{i n}^{3}}=c_{1}+c_{2} \frac{M-m_{n}}{m_{n}} \operatorname{det}\left(x_{1} \cdots x_{n-2}\right)^{2} \quad \text { for } \quad i=1, \ldots, n-1 .
\end{aligned}
$$

The equations $t_{i}=t_{j}$ of (2) are trivially satisfied by direct computations, but they are not relevant in this proof.

From equations (4) we obtain that

$$
\begin{aligned}
& r_{i j}=k_{1} \quad \text { for } \quad 1 \leq i<j \leq n-1, \\
& r_{i n}=k_{2} \quad \text { for } \quad i=1, \ldots, n-1,
\end{aligned}
$$

where $k_{1}$ and $k_{2}$ are constants. Therefore, from the first equations of (5) it follows that the masses $m_{k}$ for $k=1, \ldots, n-1$ are at the vertices of a regular $(n-2)$ simplex, and from the second equations of (5) we obtain that the mass $m_{n}$ is at the barycenter of this regular $(n-2)$-simplex. Moreover, since the barycenter must be the center of mass of the $n-1$ masses located at the vertices of a regular $(n-2)$ simplex, this forces that these $n-1$ masses must be equal. Of course, the mass $m_{n}$ located at the barycenter is arbitrary. This completes the proof of the theorem.

\section{REFERENCES}

[1] Alain Albouy, Symétrie des configurations centrales de quatre corps (French, with English and French summaries), C. R. Acad. Sci. Paris Sér. I Math. 320 (1995), no. 2, 217-220. MR.1320359(95k:70023)

[2] A. Albouy, Recherches sur le problème des $n$ corps, Notes Scientifiques et Techniques du Bureau des Longitudes, Paris (1997), 78 pp.

[3] Alain Albouy and Alain Chenciner, Le problème des $n$ corps et les distances mutuelles (French), Invent. Math. 131 (1998), no. 1, 151-184, DOI 10.1007/s002220050200. MR.1489897 (98m:70017)

[4] Alain Albouy and Vadim Kaloshin, Finiteness of central configurations of five bodies in the plane, Ann. of Math. (2) 176 (2012), no. 1, 535-588, DOI 10.4007/annals.2012.176.1.10. MR2925390

[5] Alain Albouy and Jaume Llibre, Spatial central configurations for the $1+4$ body problem, Celestial mechanics (Evanston, IL, 1999), Contemp. Math., vol. 292, Amer. Math. Soc., Providence, RI, 2002, pp. 1-16, DOI 10.1090/conm/292/04914. MR1884890(2003b:70017)

[6] O. Dziobek, Ueber einen merkwürdigen Fall des Vielkörperproblems, Astron. Nach. 152 (1900), 32-46.

[7] Yusuke Hagihara, Celestial mechanics, The MIT Press, Cambridge, Mass.-London, 1970. Volume I: Dynamical principles and transformation theory. MR0478835 (57 \#18306) 
[8] Marshall Hampton and Richard Moeckel, Finiteness of relative equilibria of the fourbody problem, Invent. Math. 163 (2006), no. 2, 289-312, DOI 10.1007/s00222-005-0461-0. MR2207019 (2008c:70019)

[9] J.L. Lagrange, Essai sur le problème des trois corps, recueil des pièces qui ont remporté le prix de l'Académie Royale des Sciences de Paris, tome IX, 1772, reprinted in Ouvres, Vol. 6 (Gauthier-Villars, Paris, 1873), 229-324.

[10] Richard Moeckel, On central configurations, Math. Z. 205 (1990), no. 4, 499-517, DOI 10.1007/BF02571259. MR1082871 (92b:70012)

[11] Richard Moeckel, Generic finiteness for Dziobek configurations, Trans. Amer. Math. Soc. 353 (2001), no. 11, 4673-4686 (electronic), DOI 10.1090/S0002-9947-01-02828-8. MR.1851188 (2002m:70018)

[12] Kenneth R. Meyer, Bifurcation of a central configuration, Celestial Mech. 40 (1987), no. 3-4, 273-282, DOI 10.1007/BF01235844. MR938404 (90c:70017)

[13] F. R. Moulton, The straight line solutions of the problem of $n$ bodies, Ann. of Math. (2) 12 (1910), no. 1, 1-17, DOI 10.2307/2007159. MR.1503509

[14] Julian I. Palmore, Classifying relative equilibria. II, Bull. Amer. Math. Soc. 81 (1975), 489491. MR0363076 (50 \#15514)

[15] Donald G. Saari, On the role and the properties of $n$-body central configurations, Proceedings of the Sixth Conference on Mathematical Methods in Celestial Mechanics (Math. Forschungsinst., Oberwolfach, 1978), Part I, Celestial Mech. 21 (1980), no. 1, 9-20, DOI 10.1007/BF01230241. MR564603 (81a:70016)

[16] Dieter S. Schmidt, Central configurations in $\mathbf{R}^{2}$ and $\mathbf{R}^{3}$, Hamiltonian dynamical systems (Boulder, CO, 1987), Contemp. Math., vol. 81, Amer. Math. Soc., Providence, RI, 1988, pp. 59-76, DOI 10.1090/conm/081/986257. MR986257 (90d:70028)

[17] S. Smale, Topology and mechanics. II. The planar n-body problem, Invent. Math. 11 (1970), 45-64. MR0321138 (47 \#9671)

[18] Aurel Wintner, The Analytical Foundations of Celestial Mechanics, Princeton Mathematical Series, v. 5, Princeton University Press, Princeton, NJ, 1941. MR0005824 (3,215b)

[19] Zhihong Xia, Central configurations with many small masses, J. Differential Equations 91 (1991), no. 1, 168-179, DOI 10.1016/0022-0396(91)90137-X. MR.1106123(92d:70010)

Departament de Matemàtiques, Universitat Autònoma de Barcelona, 08193 Bellaterra, Barcelona, Catalonia, Spain

E-mail address: jllibre@mat.uab.cat 